\title{
Characterising Testing Preorders for Broadcasting Distributed Systems ${ }^{\star}$
}

\author{
Andrea Cerone ${ }^{1}$ and Matthew Hennessy ${ }^{2}$ \\ ${ }^{1}$ IMDEA Software Institute, ${ }^{2}$ Trinity College Dublin \\ Andrea.Cerone@imdea.org, Matthew.Hennessy@scss.tcd.ie
}

\begin{abstract}
We present a process calculus for both specifying the desired behaviour of distributed systems and for describing their actual implementation; the calculus is aimed at the internet layer of the TCP/IP reference model. This allows us to define behavioural preorders in the style of DeNicola and Hennessy, relating specifications and implementations for distributed systems at this level of abstraction. The main result of the paper is a complete characterisation of these preorders, for a large class of systems, in terms of traces of extensional actions. This result underpins a sound and complete proof methodology which is demonstrated by the verification of the correct behaviour of a virtual shared memory protocol.
\end{abstract}

\section{Introduction}

Different approaches have been made to the problem of verifying the behaviour of distributed systems. This includes model checking [12/6 15] and process calculi $18 \mid 10[8] 17$ 13. In the latter a language, or calculus, is designed for both specifying desired behaviour, for example of a distributed system, and describing the proposed implementation. Verification then consists of formally proving that the two descriptions are behaviourally equivalent. The success of this approach is dependent not only on having a robust notion of behavioural equivalence, but also a high-level characterisation of the equivalence which can be used for verification purposes.

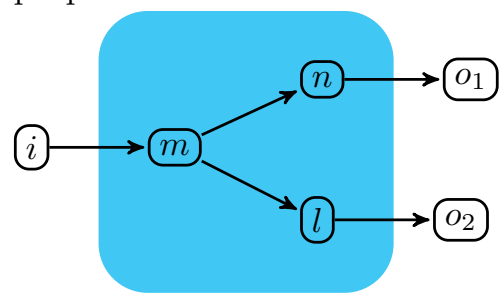

We define a process calculus for modelling (distributed) systems at a high level of abstraction, roughly at the level of the Internet Layer of the TCP/IP reference model [20]. We assume non-blocking broadcast communication between independent computational entities, although the introduction of pointto-point communication would not invalidate our results. A typical system may be seen to the left. It consists of a number of nodes or stations at which code is executed by independent processes, with an accessibility relation between the nodes. So in this example only stations $n$ and $l$ are in the range of broadcasts

\footnotetext{
* Supported by SFI project SFI 06 IN.1 1898.
} 
from station $m$ while $o_{2}$ is the only station which can pick up messages broadcast from station $l$. Thus in general communication is multicast, in that messages can be received by multiple entities simultaneously; for example messages transmitted from $m$ can be picked up at both $n$ and $l$ simultaneously. However either of the nodes $n, l$, or indeed both, can choose to ignore messages, or more generally may be in a state where broadcasts cannot currently be received. Thus broadcasts are non-blocking in that messages can be transmitted from $m$ regardless of whether or not anybody is currently listening at $n$ or $l$.

There are two kinds of nodes. The first, the internal nodes, are those at which code is executing, broadcasting and receiving messages; in our example these are $m, n$ and $l$. Here, and throughout the rest of the paper, we use shadowing to represent these internal nodes. The second are interface nodes, such as $i, o_{1}$ and $o_{2}$, which are not executing any code; however they can be used either to test the internal behaviour of the system by placing test code there, or more generally combining smaller systems to construct larger ones.

More formally, a system $\mathcal{M}$ is a pair of the form $\Gamma \triangleright M$, where $\Gamma$ is a directed graph describing the accessibility relation between nodes, or the system topology, and $M$ is a system term from a process calculus which describes the code running at the internal nodes. In this paper we focus on a sub-calculus of that of 32 in which the station code is non-probabilistic. From these papers we also borrow the (non-trivial) partial operator $\mathcal{M} \gg \mathcal{N}$ for composing systems to form larger ones. For our purposes, we focus on a relevant, large class of composable systems, which do not allow connections between interface nodes. It can be shown that all composable systems can be generated by atomic systems, which contain only one node, using the operator $¥$. As explained in 32 , the composite system $\mathcal{M} \sharp \mathcal{N}$ may be viewed as $\mathcal{N}$ extending $\mathcal{M}$ by adding new stations and adding code to execute at the interface nodes of $\mathcal{M}$; it may also be viewed as $\mathcal{N}$ blackbox testing the system $\mathcal{M}$ by placing probing code at its interface.

This compositional view of systems leads in a natural way to an adaptation of the standard testing based preorders [7] which we denote by $\mathcal{M} \sqsubseteq$ may $\mathcal{N}$ and $\mathcal{M} \sqsubseteq$ must $\mathcal{N}$. Intuitively the former means that if the application of any blackbox test $\mathcal{T}$, represented by the composite system $\mathcal{M} \ngtr \mathcal{T}$, can lead to a success, then so can the application to $\mathcal{N}$; the latter, $\mathcal{M} \sqsubseteq$ must $\mathcal{N}$, on the other hand is determined by the tested processes $\mathcal{M}, \mathcal{N}$ guaranteeing success when probed by any testing system $\mathcal{T}$.

Testing preorders can be used to capture the concept of refinement; a system $\mathcal{M}$ corresponds to an implementation of a more abstract system $\mathcal{N}$, known as the specification, if $\mathcal{M} \sqsubseteq$ may $\mathcal{N}$ and $\mathcal{M} \sqsubseteq_{\text {must }} \mathcal{N}$. However the usefulness of this notion of refinement depends on our ability to reason about these preorders. This is the topic of this paper.

In standard process calculi, such as CCS and CSP, testing preorders can be characterised by the traces, that is sequences of actions, which processes can perform, together with so-called acceptances or failures. Here however the situation is much more complicated. For example messages are broadcast asynchronously; in this respect the approach in [8] is of help. But with distributed systems we 
also have the problem of whether a multicast, that is the simultaneous broadcast of a message to multiple nodes, can be distinguished from a series of individual broadcasts to each of the nodes in turn. More importantly the ability of tests to interrogate the behaviour of a system depends on the distribution of its nodes, the accessibility between them and the accessibility to these internal nodes from the interface.

In this paper we isolate a set of extensional actions for systems; their definition is independent of the distribution of the internal nodes in the system and depends only on their accessibility from the interface nodes. They also take into account the multicasts which systems can perform, represented by the set of interface nodes which can receive the broadcast of a value at any point in time.

Traces of such extensional actions provide a sound proof method for the preorder $\sqsubseteq$ may.

Further, for those systems which do not exhibit non-terminating behaviour when considered in isolation, so-called strongly-convergent systems, it is sufficient to record deadlocks into traces to obtain a sound proof method for the preorder $\sqsubseteq$ must. As a further contribution, we show that our proof methods are complete; specifically

(1) extensional traces are complete for $\sqsubseteq$ may for arbitrary systems

(2) deadlock extensional traces are complete for $\sqsubseteq$ must when restricted to strongly convergent systems which are in addition finite spanning, meaning they can reach a finite number of states by performing a transition.

In this extended abstract we concentrate on outlining the proof of (2) which relies on exhibiting characteristic tests for (deadlock) traces of extensional actions. The definition of such tests is non-trivial; one of the main challenges when defining the characteristic test of a trace is that of coordinating the independent activities of its nodes.

As a sample application of our proof techniques, we prove the correctness of a simple virtual shared memory implementation.

The remainder of the paper is organised as follows. The calculus is presented in $\S 2$. In $\S 3$ we recall the theory of composition for networks of [3], upon which we define the testing preorders $\sqsubseteq$ may, $\sqsubseteq$ must. In $\$ 4$ we explain the extensional semantics, and define both the sets of traces and deadlock traces of systems. These are used to determine our characterisations for both testing preorders in \$5. where we put an emphasis on the completeness result for $\sqsubseteq$ must. An application of the resulting proof techniques is given in $\$ 6$, in which we prove the correctness of a virtual shared memory. We end the paper with a brief comparison with related work, in $\$ 7$.

Due to the lack of space, the technical details of $\$ 5$ and $\$ 6$ are covered in an extended version of the paper [4. A thorough discussion of the topics treated in this paper is given in [2], to which the reader is referred for detailed proofs of the results and further applications of the proof methods to real world scenarios. 


\section{A calculus for Distributed Systems}

Syntax. A directed connectivity graph $\Gamma=\left\langle\Gamma_{V}, \Gamma_{E}\right\rangle$ consists of a set of vertices $\Gamma_{V}$, representing the set of nodes of a distributed system, and a relation $\Gamma_{E} \subseteq$ $\left(\Gamma_{V} \times \Gamma_{V}\right)$ containing the connections between nodes. If $(m, n) \in \Gamma_{E}$ then node $n$ can receive messages broadcast by $m$. Given $\Gamma=\left\langle\Gamma_{V}, \Gamma_{E}\right\rangle$, we use $\Gamma \vdash m$ for $m \in \Gamma_{V}, \Gamma \vdash m \rightarrow n$ for $(m, n) \in \Gamma_{E}$ and $\Gamma \vdash m \not \rightarrow n$ for its negation.

A distributed system is modelled as a tuple $\Gamma \triangleright M$, where $\Gamma$ is a connectivity graph and $M$ is a system term which associates processes to nodes, generated by the grammar below.

$$
M, N::=\mathbf{0}|n \llbracket P \rrbracket|(M \mid N)
$$

A (system) term $M$ is a collection of sub-terms of the form $m \llbracket P \rrbracket$, which binds process $P$ to node $m$; the term $\mathbf{0}$ corresponds to the system term in which no nodes are executing processes. The code for processes will be presented shortly. We will often use the metavariables $\mathcal{M}, \mathcal{N}, \cdots$ when referring to a system $\Gamma \triangleright M$.

Let nodes $(M)$ be the set of the node names appearing in $M$. We only consider the sublanguage of well-formed systems $\Gamma \triangleright M$ such that nodes $(M) \subseteq \Gamma_{V}$, and such that each node name occurs at most once in $M$. These constraints ensure that in systems, nodes running code are part of the system topology, and no node can be bound to multiple processes. It is possible for nodes appearing in $\Gamma$ not to occur in $M$. At least intuitively, such nodes represent the external environment of a system and will be used to test its behaviour (\$3). The set of the external nodes of a system $\Gamma \triangleright M$, formally defined as $\Gamma_{V} \backslash \operatorname{nodes}(M)$, is called the interface of the system and denoted by $\operatorname{Intf}(\Gamma \triangleright M)$.

The syntax for processes, given below, is a straightforward instance of a standard process calculus, and their constructs should be self explanatory. Here we assume a (at most countable) set of process definitions of the form $A \Leftarrow P$.

$$
P, Q::=\mathbf{0}|c !\langle e\rangle . P| c ?(x) . P|\tau . P| \omega|P+Q| \text { if } b \text { then } P \text { else } Q \mid A(\tilde{x})
$$

For processes we assume some language for Boolean expressions, $b, b^{\prime}, \ldots$ which includes variables, $x, y, \cdots$ and the Boolean values, $\{$ true, false $\}$. In the standard manner we assume an interpretation function $\llbracket \cdot \rrbracket$ which maps all closed Boolean expressions to some Boolean value. In a similar manner we assume another language of (value) expressions, $e, e^{\prime}, \cdots$, which again may contain variables, and values $v, w, \cdots$ from some finite value set; this language also comes equipped with an interpretation function, mapping each closed expression into one of the finite set of values.

We also assume a special clause $\omega$ which will be used for testing purposes in \$3. Any system which has no occurrence of the special clause is called proper.

Intensional Semantics. We define a collection of Structured Operational Semantics rules, whose judgements take the form $(\Gamma \triangleright M) \stackrel{\mu}{\longrightarrow}(\Gamma \triangleright N)$, to define the behaviour of systems. The action $\mu$ can have either the form (a) m.c! $v$, node 


$$
\begin{aligned}
& \text { (B-BROAD) } \frac{P \stackrel{c ! v}{\longrightarrow} Q}{\Gamma \triangleright n \llbracket P \rrbracket \stackrel{n . c ! v}{\longrightarrow} \Gamma \triangleright n \llbracket Q \rrbracket} \\
& \text { (в-REC) } \frac{P \stackrel{c ? v}{\longrightarrow} Q \quad \Gamma \vdash m \rightarrow n}{\Gamma \triangleright n \llbracket P \rrbracket \stackrel{m . c ? v}{\longrightarrow} \Gamma \triangleright n \llbracket Q \rrbracket} \\
& \text { (B-DEAF) } \frac{P \stackrel{c ? v}{\longrightarrow}}{\Gamma \triangleright n \llbracket P \rrbracket \stackrel{m . c ? v}{\longrightarrow} \Gamma \triangleright n \llbracket P \rrbracket} \\
& \text { (в-DISC) } \frac{\Gamma \vdash m \nrightarrow \rightarrow n}{\Gamma \triangleright n \llbracket P \rrbracket \stackrel{m . c ? v}{\longrightarrow} \Gamma \triangleright n \llbracket P \rrbracket} \\
& \text { (в- 0) } \overline{\Gamma \triangleright \mathbf{0} \stackrel{m . c ? v}{\longrightarrow} \Gamma \triangleright \mathbf{0}} \\
& \text { (в- } \tau) \frac{P \stackrel{\tau}{\longrightarrow} Q}{\Gamma \triangleright n \llbracket P \rrbracket \stackrel{\tau}{\longrightarrow} \Gamma \triangleright n \llbracket Q \rrbracket} \\
& (\mathrm{B}-\tau . \mathrm{PROP}-\mathrm{L}) \frac{\Gamma \triangleright M \stackrel{\tau}{\longrightarrow} \Gamma \triangleright L}{\Gamma \triangleright M|N \stackrel{\tau}{\longrightarrow} \Gamma \triangleright L| N} \\
& \text { (B- } \tau \text {.PROP }-\mathrm{R}) \frac{\Gamma \triangleright N \stackrel{\tau}{\longrightarrow} \Gamma \triangleright L}{\Gamma \triangleright M|N \stackrel{\tau}{\longrightarrow} \Gamma \triangleright M| L} \\
& \text { (в-SYNC) } \frac{\Gamma \triangleright M \stackrel{\mu_{1}}{\longrightarrow} \Gamma \triangleright M^{\prime} \Gamma \triangleright N \stackrel{\mu_{2}}{\longrightarrow} \Gamma \triangleright N^{\prime}}{\Gamma \triangleright M\left|N \stackrel{\left(\mu_{1} \circ \mu_{2}\right)}{\longrightarrow} \Gamma \triangleright M^{\prime}\right| N^{\prime}} \\
& \begin{array}{|c|l|l|}
\hline \mu_{1} \circ \mu_{2} & m . c ! v & m . c ? v \\
\hline m . c ! v & & m . c ! v \\
\hline m . c ? v & m . c ! v & m . c ? v \\
\hline
\end{array}
\end{aligned}
$$

Fig. 1. Labelled Transition Semantics for (high level) systems

$$
\begin{aligned}
& (\mathrm{s}-\mathrm{SND}) \frac{\llbracket e \rrbracket=v}{c !\langle e\rangle \cdot P \stackrel{c ! v}{\longrightarrow} P} \quad \quad \text { (s-RCv) } \frac{}{c ?(x) \cdot P \stackrel{c ? v}{\longrightarrow}\{v / x\} P} \\
& \text { (s- } \tau) \frac{\text { (s-Sum-L) }}{\tau . P \stackrel{(\tau)}{\longrightarrow} P} \stackrel{\stackrel{\alpha}{\longrightarrow} P^{\prime}}{P+Q \stackrel{\alpha}{\longrightarrow} P^{\prime}} \\
& \text { (s-then) } \frac{P \stackrel{\alpha}{\longrightarrow} P^{\prime} \quad \llbracket b \rrbracket=\text { true }}{\text { if } b \text { then } P \text { else } Q \stackrel{\alpha}{\longrightarrow} P^{\prime}} \quad \text { (s-Pdef) } \frac{A(\tilde{x}) \Leftarrow P \quad\{\tilde{e} / \tilde{x}\} P \stackrel{\alpha}{\longrightarrow} Q}{A\langle\tilde{e}\rangle \stackrel{\alpha}{\longrightarrow} Q}
\end{aligned}
$$

Fig. 2. Pre-semantics of processes

$m$ broadcasts value $v$ along channel $c$, (b) $m . c ? v$, the system receives a broadcast of value $v$ along channel $c$ from the external node $m$, or (c) $\tau$, some node performs an internal activity.

The rules of the labelled transition semantics are depicted in Figure 1 . They are based on a pre-semantics for processes, whose rules are given in Figure 2 and should be self-explanatory. Some symmetric rules have been omitted.

Rule (B-BROAD) models a node which is willing to transmit a value $v$, while the next four rules deal with how stations react to such a broadcast. When a station is within the transmitter's range and is waiting to detect a value along channel $c$, it will receive it correctly (Rule (B-REC)). On the other hand, if either the station is not waiting to detect a value along channel $c$ (Rule (B-DEAF)) or is not in the sender's transmission range (rule (B-DISC)), then the broadcast is ignored. In rule (B-DEAF), $P \stackrel{c ? p}{\longrightarrow}$ means that $P \stackrel{c ? v}{\longrightarrow} Q$ for no process $Q$. Finally, the $\mathbf{0}$ system term ignores all transmissions (Rule (B- $\mathbf{0})$ ).

Rule (B-SYNC) models how nodes interact. The action performed by a system $(\Gamma \triangleright M \mid N)$ is determined by the individual actions performed by $\Gamma \triangleright M$ and 
$\Gamma \triangleright N$, according to a (partial) binary operator for actions $\circ$ defined at the right of Rule (B-SYNC) in Figure 1. Here it is important to note that the action induced by a transmission and a reception is again a transmission, thus implementing broadcast communication; see [18 for a detailed discussion. The remaining rules, modelling internal activity, are straightforward.

Example 1. Consider the system $\mathcal{M}=\Gamma \triangleright M$, where $\Gamma$ is the directed graph depicted on page $1, M$ is $m \llbracket A \rrbracket|n \llbracket A \rrbracket| l \llbracket A \rrbracket$ and $A \Leftarrow c ?(x) . c !\langle x\rangle$. All the internal nodes $m, n, l$ are waiting to receive a value via channel $c$ in $\mathcal{M}$; once any of these nodes has received such a value, it will forward it along the same channel. One possible behaviour of $\mathcal{M}$ can be summarised as follows; first node $m$ detects a broadcast of an arbitrary value $v$ along channel $c$, performed by node $i$. Nodes $n, l$ are not affected by this broadcast, since they are not in the range of transmission of $i$. Using Rules (B-REC), (B-DISC) and (B-SYNCH) we can infer the transition $\mathcal{M} \stackrel{i . c ? v}{\longrightarrow} \mathcal{M}_{1}$, where $\mathcal{M}_{1}=\Gamma \triangleright m \llbracket P_{v} \rrbracket|n \llbracket A \rrbracket| l \llbracket A \rrbracket$ and $P_{v}=c !\langle v\rangle$.

Next node $m$ forwards value $v$ to both nodes $n$ and $l$, which are in its range of transmission. This is formalised by the transition $\mathcal{M}_{1} \stackrel{m . c ! v}{\longrightarrow} \mathcal{M}_{2}$, where $\mathcal{M}_{2}=$ $\Gamma \triangleright m \llbracket \mathbf{0} \rrbracket\left|n \llbracket P_{v} \rrbracket\right| l \llbracket P_{v} \rrbracket$, which is obtained using rules (B-BROAD), (B-REC) and (B-SYNCH). Finally, we have a broadcast fired by $n$ followed by one fired by $l$. Let $\mathcal{M}_{3}=\Gamma \triangleright m \llbracket \mathbf{0} \rrbracket|n \llbracket \mathbf{0} \rrbracket| l \llbracket P_{v} \rrbracket$ and $\mathcal{M}_{4}=\Gamma \triangleright m \llbracket \mathbf{0} \rrbracket|n \llbracket \mathbf{0} \rrbracket| l \llbracket \mathbf{0} \rrbracket$; then we have $\mathcal{M}_{2} \stackrel{n . c ! v}{\longrightarrow} \mathcal{M}_{3} \stackrel{l . c ! v}{\longrightarrow} \mathcal{M}_{4}$.

Reduction Semantics. In the following we will want to discuss the behaviour of systems when isolated from its interface; that is, when input actions of interface nodes are inhibited. To this end, we define a reduction relation $\rightarrow$ by letting $\mathcal{M} \rightarrow \mathcal{N}$ if either $\mathcal{M} \stackrel{m . c ! v}{\longrightarrow} \mathcal{N}$ or $\mathcal{M} \stackrel{\tau}{\longrightarrow} \mathcal{N}$; any maximal sequence of reductions rooted in a system $\mathcal{M}$ is called a computation for $\mathcal{M}$. Note that reductions $\rightarrow$ can be defined directly via a reduction semantics; see [2], $§ 2.2$ and $\S 2.4$.

\section{$3 \quad$ Testing Distributed Systems}

Extension of Systems Following the approach of [3] we focus on a specific class of systems and how they can be extended to obtain larger systems.

Definition 1 (Composable Systems). A system $\mathcal{M}=\Gamma \triangleright M$ is composable if whenever $\Gamma \vdash m \rightarrow n$ then either $m \in \operatorname{nodes}(M)$ or $n \in \operatorname{nodes}(M)$.

Henceforth we will always assume that systems are composable. Such systems do not allow connections between nodes in their interface; from the point of view of a system, the only visible information about its external environment consists of the set of access points to the system. In our terminology its interface.

Focusing on composable systems is not restrictive, in that connections between external nodes do not affect the behaviour of systems, so that any system $\mathcal{M}$ can be reduced to a composable one $\mathcal{M}^{\prime}$ without affecting the transitions it can perform. Below we define an extension operator which allows us to infer a larger system from a given one, in which nodes which were external in the latter may now be connected. 

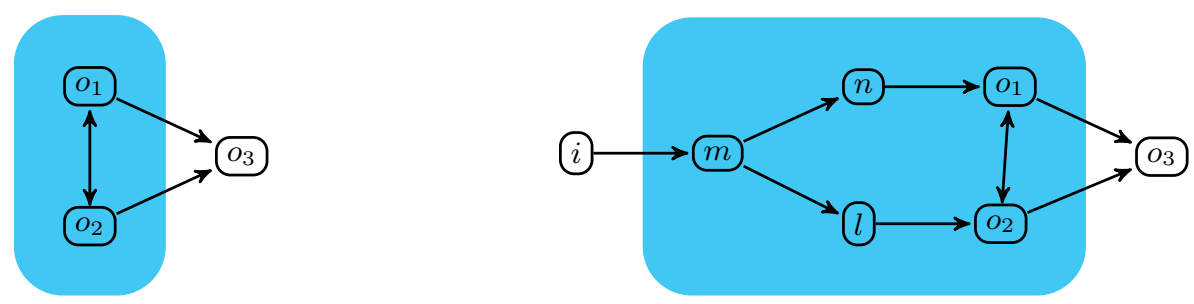

Fig. 3. Two systems $\mathcal{N}$ and $\mathcal{L}=(\mathcal{M} \ngtr \mathcal{N})$.

Definition 2 (Systems Extension). Let $\mathcal{M}=\left(\Gamma_{M} \triangleright M\right), \mathcal{N}=\left(\Gamma_{N} \triangleright N\right)$ be (composable) systems; the extension of $\mathcal{M}$ with $\mathcal{N},(\mathcal{M} \sharp \mathcal{N})$, is defined whenever nodes $(M) \cap\left(\Gamma_{N}\right)_{V}=\emptyset$, and it is equivalent to $\left(\Gamma_{M} \cup \Gamma_{N}\right) \triangleright(M \mid N)$, where $\Gamma_{M} \cup \Gamma_{N}$ is defined as the pointwise union of their sets of vertices and edges.

Intuitively, $\mathcal{M} \gg \mathcal{N}$ describes an extension of the system $\mathcal{M}$, where the information about its external environment is supplied by a second system $\mathcal{N}$; such system can contain the code run by interface nodes of $\mathcal{M}$, and the connections between its interface nodes. But it can also contain new nodes, which did not appear in $\mathcal{M}$. Also, in the composite system, the topological structure of $\mathcal{M}$ is left unchanged; this property is desirable, as we wish to use the operator $\$$ to implement blackbox testing. In [3] we proved that $\$$ is associative and closed with respect to the set of composable systems, and that it is the most expressive operator which can be used to implement blackbox testing.

Example 2. Let $\mathcal{M}$ be the system of Example1 its interface $\operatorname{Intf}(\mathcal{M})=\left\{i, o_{1}, o_{2}\right\}$ represents its external environment. By using the extension operator $\$$ we can obtain a new system which contains $\mathcal{M}$, and which also gives new information about the connections of the nodes in the external environment of $\mathcal{M}$.

For example, consider the system $\mathcal{N}=\Gamma_{N} \triangleright o_{1} \llbracket A \rrbracket \mid o_{2} \llbracket A \rrbracket$, depicted on the left of Figure 3, here $A$ is as defined in Example 1. This system specifies the code nodes $o_{1}, O_{2}$ are running, together with their connections; it also has a fresh node $o_{3}$ in its own interface. The composite system $\mathcal{L}=(\mathcal{M} \sharp \mathcal{N})$ is well-defined, and it models the system obtained by extending $\mathcal{M}$ with the information regarding its external environment provided by $\mathcal{N}$. The system $\mathcal{L}$ is depicted to the right of Figure 3 . Here the sub-system $\mathcal{N}$ could be viewed as a black box tester for the original system $\mathcal{M}$, placing probing code at two of $\mathcal{M}$ 's interface nodes, and having another node $o_{3}$ where the results of this probing could be collected.

Testing Preorders. We say that a system $\mathcal{M}$ is successful if the clause $\omega$ appears unguarded in the code of one of its nodes, while a computation of a system $\mathcal{M}$ is said to be successful if it contains a successful system. Given two systems $\mathcal{M}, \mathcal{T}$ we say that $\mathcal{M}$ may-pass $\mathcal{T}$ if $\mathcal{M} \sharp \mathcal{T}$ has a successful computation, while $\mathcal{M}$ must-pass $\mathcal{T}$ if all its computations are successful. 
Definition 3 (Testing Preorders). Let $\mathcal{M}, \mathcal{N}$ be two systems; we say that $\mathcal{M} \sqsubseteq$ may $\mathcal{N}$ if for any $\mathcal{T}$ which can be used to extend both $\mathcal{M}, \mathcal{N}$ we have that $\mathcal{M}$ may-pass $\mathcal{T}$ implies $\mathcal{N}$ may-pass $\mathcal{T}$. Similarly, we define $\mathcal{M} \sqsubseteq$ must $\mathcal{N}$ in the same way, this time using the must-pass testing relation. We say that $\mathcal{M} \sqsubseteq \mathcal{N}$ if both $\mathcal{M} \sqsubseteq_{\text {may }} \mathcal{N}$ and $\mathcal{M} \sqsubseteq_{\text {must }} \mathcal{N}$ are true; finally, we use the notation $\mathcal{M} \simeq \mathcal{N}$ if both $\mathcal{M} \sqsubseteq \mathcal{N}$ and $\mathcal{N} \sqsubseteq \mathcal{M}$ are true.

Example 3 (Deadlocks). Let $\Gamma$ represent the system topology containing two nodes $m, e$ and having as its only connections $\Gamma \vdash m \rightarrow e, e \rightarrow m$. Let $\mathcal{M}=$ $\Gamma \triangleright m \llbracket P \rrbracket, \mathcal{N}=\Gamma \triangleright m \llbracket Q \rrbracket$, where $P=c !\langle v\rangle, Q=c ?(x) . c !\langle v\rangle$. Both systems have the ability to broadcast value $v$ along channel $c$; however, in $\mathcal{N}$ this broadcast is enabled only after a broadcast along channel $c$ has been performed by node $e$. That is, $\mathcal{N} \rightarrow \mathcal{N}^{\prime}$ for no $\mathcal{N}^{\prime}$.

These two systems can be distinguished via the must-pass testing relation by the test $\mathcal{T}=\Gamma_{e} \triangleright e \llbracket c ?(x) . \omega \rrbracket$; here $\Gamma_{e}$ is the graph with the single node $e$. Note that $\mathcal{M}$ must-pass $\mathcal{T}$, while $\mathcal{N}$ must-pass $\mathcal{T}$ is not true. Therefore $\mathcal{M} \unrhd_{\text {must }} \mathcal{N}$.

It is also possible to exhibit a test which $\mathcal{N}$ must-passes, but $\mathcal{M}$ does not. To this end, let $\mathcal{T}^{\prime}=\Gamma_{e} \triangleright e \llbracket c ?(x) . \mathbf{0}+\tau . \omega \rrbracket$. It is easy to see that $\mathcal{M} \gg \mathcal{T}^{\prime}$ has an unsuccessful computation; however, since $\mathcal{N}$ is deadlocked and cannot broadcast a value, the only possibility for $\mathcal{N} \sharp \mathcal{T}^{\prime}$ is that the testing component performs a $\tau$ action, thus entering a successful state. $\mathcal{N}$ must-pass $\mathcal{T}^{\prime}$, hence $\mathcal{N} \unrhd_{\text {must }} \mathcal{M}$.

What distinguishes $\mathcal{M}$ from $\mathcal{N}$ is that the latter cannot broadcast value $v$ without first receiving a value from node $e$ first, that is it is deadlocked. However suppose that we add the possibility to $\mathcal{N}$ to directly broadcast value $v$, leading to the system $\mathcal{N}^{\prime}=\Gamma \triangleright m \llbracket Q^{\prime} \rrbracket$, where $Q^{\prime}=Q+c !\langle v\rangle$; as we will see, it is impossible to distinguish $\mathcal{N}^{\prime}$ from $\mathcal{M}$. Note that $\mathcal{N}^{\prime}$ is not deadlocked.

Also, note that $P, Q^{\prime}$ are valid value-passing CCS processes, and that their sets of acceptances are different. In fact, in value-passing CCS, $P$ must-passes the test $c !\langle v\rangle+\tau . \omega$, but the same is not true for $Q^{\prime}$; also, $Q^{\prime}$ must-passes the test $c !\langle v\rangle . \omega$, but this is not true for $P$. This example gives an intuition that deadlocks, rather than acceptances, should be taken into account when giving a characterisation of the preorder $\sqsubseteq_{\text {must }}$.

\section{Extensional Semantics}

The extensional semantics of systems is defined in Figure 44 its transitions $\mathcal{M} \stackrel{\lambda}{\longmapsto} \mathcal{N}$ can be either (a) internal activities $\tau$, (b) inputs $i . c$ ? $v$ performed by an input node $i$ or (c) broadcast actions $c ! v \triangleright \eta$, observable at a non-empty set of output nodes $\eta$. Note that any node name mentioned in the action of the transition occurs in the interface of the source configuration $\mathcal{M}$. The set of extensional actions is denoted by EAct $_{\tau}$, while EAct $=$ EAct $_{\tau} \backslash\{\tau\}$.

Rules (s-TAU) and (s-SHH) model unobservable activities. The first rule propagates internal activities of nodes to systems, while the second rule states that broadcasts which cannot be detected by any external node of a system cannot be observed. Rule (S-IN) propagates input actions to the extensional semantics; 


$$
\begin{aligned}
& \text { (s-TAU) } \frac{\mathcal{M} \stackrel{m . \tau}{\longrightarrow} \mathcal{N}}{\mathcal{M} \stackrel{\tau}{\longrightarrow} \mathcal{N}} \quad \text { (s-ShH) } \frac{\mathcal{M} \stackrel{m . c ! v}{\longrightarrow} \mathcal{N}}{\mathcal{M} \stackrel{\tau}{\longrightarrow} \mathcal{N}} \quad\{n \in \operatorname{lntf}(\mathcal{M}) \mid \mathcal{M} \vdash m \rightarrow n\}=\emptyset \\
& \text { (s-IN) } \frac{\mathcal{M} \stackrel{i . c ? v}{\longrightarrow} \mathcal{N}}{\mathcal{M} \stackrel{i . c ? v}{\longrightarrow} \mathcal{N}} \quad \text { (s-OuT) } \frac{\mathcal{M} \stackrel{m . c ! v}{\longrightarrow} \mathcal{N}}{\mathcal{M} \stackrel{c ! v \triangleright \eta}{\longrightarrow} \mathcal{N}} \quad \eta:=\{n \in \operatorname{lntf}(\mathcal{M}) \mid \mathcal{M} \vdash m \rightarrow n\} \neq \emptyset
\end{aligned}
$$

Fig. 4. Extensional Semantics

finally, Rule (S-OUT) models outputs which can be observed by a set of external nodes $\eta$.

The extensional semantics endows systems with the structure of a LTS [14], which we call the extensional LTS of systems. Most of the terminology used for LTSs in the literature can be then readapted to systems. However our definition of weak extensional actions, taken from [3] needs to be non-standard.

Definition 4 (Weak Extensional Actions). For any systems $\mathcal{M}, \mathcal{N}$, we say that $\mathcal{M} \stackrel{\tau}{\longmapsto} \mathcal{N}$ if $\mathcal{M} \stackrel{\tau}{\longmapsto}{ }^{*} \mathcal{N}$; here $\stackrel{\tau}{\longmapsto}^{*}$ is the reflexive, transitive closure of $\stackrel{\tau}{\longmapsto}$. Further, we say that $\mathcal{M} \stackrel{i . c ? v}{\Longrightarrow} \mathcal{N}$ if $\mathcal{M} \stackrel{\tau}{\Longrightarrow} \stackrel{i . c ? v}{\longmapsto} \stackrel{\tau}{\Longrightarrow} \mathcal{N}$. Finally,

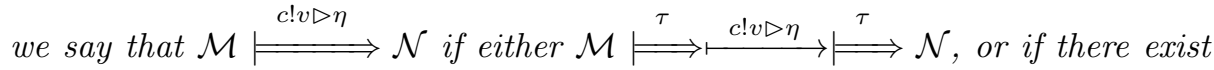
two non-empty sets of nodes $\eta_{1}, \eta_{2}$ such that $\eta_{1} \cup \eta_{2}=\eta, \eta_{1} \cap \eta_{2}=\emptyset$ and $\mathcal{M} \stackrel{c ! v \triangleright \eta_{1}}{\rightleftharpoons} \stackrel{c ! v \triangleright \eta_{2}}{\rightleftharpoons} \mathcal{N}$.

These single weak transitions are extended to sequences $\mathcal{M} \longmapsto$ $s \in E A c t^{*}$, in the obvious manner.

The complication in the definition of $\mathcal{M} \stackrel{c ! v \triangleright \eta}{\rightleftharpoons} \mathcal{N}$ is necessary in order to be able to simulate a multicast $c ! v \triangleright \eta$, where the set $\eta$ contains more than one node name, by a sequence of single broadcasts; this is shown in the Example below.

Example 4. Let $\mathcal{M}=\Gamma_{M} \triangleright m \llbracket c ! v \rrbracket$, where $\Gamma_{M} \vdash m \rightarrow o_{1}, m \rightarrow o_{2}$. Also, let $\mathcal{N}=\Gamma_{N} \triangleright m \llbracket c ! v \rrbracket \mid n \llbracket c ! v \rrbracket$, where $\Gamma_{N} \vdash m \rightarrow o_{1}, n \rightarrow o_{2}$. Both systems are able to deliver value $v$, along channel $c$, to the interface nodes $o_{1}, o_{2}$. However, while $\mathcal{M}$ does it with a single broadcast, $\mathcal{N}$ uses two broadcasts which can be detected by nodes $o_{1}, o_{2}$ individually.

One could expect that there is a test that allows us to distinguish $\mathcal{M}$ from $\mathcal{N}$, in the sense of $\sqsubseteq_{\text {may }}$. However, at least intuitively, the broadcast of $\mathcal{M}$ can be simulated in system $\mathcal{N}$, by firing the two broadcasts in sequence. That is, the action $\mathcal{M} \stackrel{c ! v \triangleright\left\{o_{1}, o_{2}\right\}}{\Longrightarrow}$ can be matched by another action $\mathcal{N} \stackrel{c ! v \triangleright\left\{o_{1}, o_{2}\right\}}{=}$, where the latter can be inferred from $\mathcal{N} \stackrel{c ! v \triangleright\left\{o_{1}\right\}}{\rightleftharpoons} \stackrel{c ! v \triangleright\left\{o_{2}\right\}}{\rightleftharpoons}$ using our nonstandard definition of weak extensional actions.

If a test $\mathcal{T}$ is used to test the system $\mathcal{M}$, each configuration $\mathcal{T}^{\prime}$ reached by the test $\mathcal{T}$ after $\mathcal{M}$ has broadcast value $v$, can be also obtained when $\mathcal{T}$ is used 
to test the system $\mathcal{N}$, by letting the latter fire its two broadcasts in sequence. That is, $\mathcal{M} \sqsubseteq$ may $\mathcal{N}$.

On the other hand, $\mathcal{N} \nsubseteq_{\text {may }} \mathcal{M}$. To prove this, it is sufficient to provide a test $\mathcal{T}$ which $\mathcal{N}$ may-passes, but $\mathcal{M}$ does not. The reader can easily check that $\Gamma_{T} \triangleright o_{1} \llbracket c ?(x) . c !\langle w\rangle \rrbracket \mid o_{2} \llbracket c ?(x) . c ?(y)$.if $(x=y)$ then $\mathbf{0}$ else $\omega \rrbracket$, where $\Gamma_{T} \vdash o_{1} \rightarrow o_{2}$, is one such test.

A similar argument shows that $\mathcal{N} \sqsubseteq$ must $\mathcal{M}$, while $\mathcal{M} \nsubseteq$ must $\mathcal{N}$.

\section{Characterisation of the Testing preorders}

Traces and Deadlock Traces. A system $\mathcal{M}$ is finite spanning if any $\mathcal{N}$ in the extensional LTS generated by $\mathcal{M}$ can reach a finite number of systems by performing a transition. It is convergent if it has no infinite computation, strongly convergent if every state in the extensional LTS it generates is convergent.

From Example 3 we know that must-testing preorder is sensitive to deadlocks; this is the essential ingredient to the following definition:

Definition 5 (Traces, Deadlock Traces). Let $\delta \notin E A c t$. For any $\mathcal{M}$ we let

$$
\begin{aligned}
& \operatorname{Traces}(\mathcal{M})=\left\{s \in \text { EAct }^{*} \mid \mathcal{M} \longmapsto \mathcal{s} \longmapsto \mathcal{N} \text { for some } \mathcal{N}\right\} \\
& \operatorname{Dtraces}(\mathcal{M})=\left\{s:: \delta \mid \mathcal{M} \stackrel{s}{\Longrightarrow} \mathcal{N} \text { for some } \mathcal{N}, s \in \text { EAct }^{*} \text { such that } \mathcal{N} \nrightarrow\right\}
\end{aligned}
$$

We use the symbol :: to separate occurrences of elements in lists.

Example 5. Consider again the system $\mathcal{M}, \mathcal{N}$ of Example 3 We have already noted that $\mathcal{M} \nsubseteq$ must $\mathcal{N}$, and $\mathcal{N} \nsubseteq$ must $\mathcal{M}$. Also, we have that $\delta \in \operatorname{Dtraces}(\mathcal{N})$ (note that $\mathcal{N} \nrightarrow \triangleright$ ), while $\delta \notin \operatorname{Dtraces}(\mathcal{M})$. Further, we have that $c ! v \triangleright\{e\}:: \delta \in$ $\operatorname{Dtraces}(\mathcal{M})$, but this trace is not in $\operatorname{Dtraces}(\mathcal{N})$; in fact, in the latter system the broadcast of value $v$ can happen only after a value has been received along channel $c$. That is, $c ? w:: c ! v \triangleright\{e\}:: \delta \in \operatorname{Dtraces}(\mathcal{M})$ for an arbitrary value $w$.

Now consider the system $\mathcal{N}^{\prime}$ of Example 3. Note that $c ! v \triangleright\{e\}:: \delta \in \operatorname{Dtraces}\left(\mathcal{N}^{\prime}\right)$, and $\delta \notin \operatorname{Dtraces}\left(\mathcal{N}^{\prime}\right)$. Even more, the two systems $\mathcal{M}, \mathcal{N}^{\prime}$ share the same set of deadlock traces, the minimal fragment of which we are interested in being $\{c !\langle v\rangle:: \delta, c ?(w):: \delta \mid w$ arbitrary value $\}$. Theorem 1, coming up, implies that $\mathcal{M}$ and $\mathcal{N}^{\prime}$ cannot be distinguished by the must-pass relation.

Full Abstraction. We can now state the main result of the paper:

Theorem 1 (Characterisation of the testing preorders). Let $\mathcal{M}, \mathcal{N}$, be two proper systems. Then

(1) $\mathcal{M} \sqsubseteq$ may $\mathcal{N}$ if and only if $\operatorname{Traces}(\mathcal{M}) \subseteq \operatorname{Traces}(\mathcal{N})$,

(2) if $\mathcal{M}, \mathcal{N}$ are finite spanning and strongly convergent then $\mathcal{M} \sqsubseteq$ must $\mathcal{N}$ if and only if Dtraces $(\mathcal{M}) \supseteq \operatorname{Dtraces}(\mathcal{N})$. 
The restriction to proper systems is natural, as the special action $\omega$ should only appear in systems used for testing. The restriction to strongly convergent systems in part (2) is needed because in our definition of deadlock traces we did not take divergence into account. Doing so would be quite complicated as, due to the non-blocking nature of broadcasts, it is possible to define two divergent systems $\mathcal{M}, \mathcal{N}$ which are not must-testing related. This is in contrast with the standard theory of must-testing [7]. See 2], Remark 4.4.3, Page 88, for a detailed discussion. See also $\S 4.4 .3$, Page 98, for a potential solution to this problem.

In this extended abstract we only have space to give an outline of Theorem 1(2). This is split into two parts, soundness and completeness.

Theorem 2 (Soundness for $\sqsubseteq$ must). If Dtraces $(\mathcal{M}) \supseteq \operatorname{Dtraces}(\mathcal{N})$ then $\mathcal{M}$ $\sqsubseteq_{\text {must }} \mathcal{N}$, for proper, finite spanning, strongly convergent systems.

Proof. It suffices to show that inclusion of deadlock traces is preserved when extending systems by adding a new node. That is, if $\operatorname{Dtraces}(\mathcal{M}) \supseteq \operatorname{Dtraces}(\mathcal{N})$, and $\mathcal{T}=\Gamma_{T} \triangleright e \llbracket P \rrbracket$ is a system such that $\mathcal{M} \sharp \mathcal{T}$ and $\mathcal{N} \gg \mathcal{T}$ are defined, then $\operatorname{Dtraces}(\mathcal{M} \sharp \mathcal{T}) \supseteq \operatorname{Dtraces}(\mathcal{N} \sharp \mathcal{T})$. See [2], $\S 4.3 .1$ and $\S 4$.4.1, for a proof.

The standard approach to prove the converse to Theorem 2 is that of providing characteristic tests for deadlock traces.

Proposition 1 (Characteristic Tests). Let $\mathcal{M}$ be a proper, finite spanning, strongly convergent system. Then for any set of nodes $\eta$ such that $\operatorname{Intf}(\mathcal{M}) \subseteq \eta$ and trace $t$ there exists a system $\mathcal{T}_{t}^{\eta}$ such that $t \notin \operatorname{Dtraces}(\mathcal{M})$ if and only if $\mathcal{M}$ must-pass $\mathcal{T}_{t}^{\eta}$. We say that $\mathcal{T}_{t}^{\eta}$ is a characteristic test for $t$.

Proof. See $\S 4.3 .2$ and $\S 4.4 .2$ of [2].

Characteristic tests are parameterised by a set of nodes $\eta$; in general the topology of a test depends on the interface of the system being tested. Here we only describe the informal behaviour of the characteristics tests $\mathcal{T}_{t}^{\eta}$, while formal definitions are given in [4], $\S \mathrm{A}$.

A characteristic test $\mathcal{T}_{t}^{\eta}$ contains all the nodes in $\eta$ and a fresh node $c n$, called controller node. The controller node is connected (in both directions) with each of the nodes in $\eta$. The test $\mathcal{T}_{t}^{\eta}$ tests whether a system does not exhibit the deadlock trace $t$ by testing sequentially for every action included in $t$ (or for deadlock when testing for $\delta$ ), then declaring success whenever it determines that the current action being tested cannot be performed by the tested system.

To achieve this, in $\mathcal{T}_{t}^{\eta}$ the nodes in $\eta$ constantly report (via a fresh channel) the observed behaviour of the tested system to the controller node; these partial information are then used by the latter to infer whether the (extensional) action being tested has been performed by the tested system, in which case $\mathcal{T}_{t}^{\eta}$ proceeds by testing for the next action in $t$. In the process of inferring whether an extensional action has been performed by the tested system, the controller node also asks nodes in $\eta$ to report the absence of observed behaviour. For example, detecting an action of the form $c ! v \triangleright \eta^{\prime}$ requires that no node in $\eta \backslash \eta^{\prime}$ observed a broadcast. Or, when testing for deadlock, no node in $\eta$ should detect 
a broadcast. Below we give a detailed explanation of the protocol used by $\mathcal{T}_{t}^{\eta}$ to detect an extensional action, with a particular emphasis to an output action of the form $c ! v \triangleright \eta^{\prime}$; the protocol runs in three stages.

Detect: The controller node waits for a relevant subset of nodes in $\eta$, consisting of those nodes mentioned in the action being tested, to report to the controller node that their local contribution to the action being tested has been performed. In the case of an action of the form $c ! v \triangleright \eta^{\prime}$, node $c n$ awaits a message from each of the nodes in $\mathrm{cn}$. Each of such nodes broadcasts to the $c n$ only if it detects a broadcast of value $v$ along channel $c$.

Check: The controller node requests to all the nodes in $\eta$ whether they observed any other additional activity from the tested system, and awaits a response from each of such nodes; in contrast, the latter do not answer to such a request if they observed some activity from the tested system. In the case of an action of the form $c ! v \triangleright \eta^{\prime}$, nodes in $\eta^{\prime} \backslash \eta$ do not answer whether they detected a broadcast performed by the tested system.

Proceed: The controller node sends a request to all nodes in $\eta$ that it is ready to detect the next action in the trace being tested. If nodes in $\eta$ observe some activity performed by the testee, they deadlock, causing the execution of $\mathcal{T}^{\eta}$ to be eventually unable to proceed.

Failure: At any given point, the controller node can non-deterministically declare success, exception made for when it detects that the entire trace being tested has been performed by the testee. In this case $\mathcal{T}_{t}^{\eta}$ deadlocks, thus causing the test to fail.

Theorem 3 (Completeness for $\left.\sqsubseteq_{\text {must }}\right) . \mathcal{M} \sqsubseteq_{\text {must }} \mathcal{N}$ implies Dtraces $(\mathcal{M}) \supseteq$ Dtraces $(\mathcal{N})$, assuming both are finite spanning, strongly convergent, proper systems.

Proof. Suppose $\mathcal{M} \sqsubseteq$ must $\mathcal{N}$ is true and $t \in \operatorname{Dtraces}(\mathcal{N})$; we need to show that $t \in \operatorname{Dtraces}(\mathcal{M})$. This will follow from the previous proposition if $\mathcal{M}$ must-pass $\mathcal{T}_{t}^{\eta}$ is not true, where we choose $\eta$ to be $\operatorname{Intf}(\mathcal{M}) \cup \operatorname{Intf}(\mathcal{N})$.

But this follows form the assumption $\mathcal{M} \sqsubseteq$ must $\mathcal{N}$ because again the previous proposition gives that $\mathcal{N}$ must-pass $\mathcal{T}_{t}^{\eta}$ is false.

\section{Application: Virtual Shared Memory}

To show the usefulness of our results, we prove the correctness of a Virtual Shared Memory (VSM) protocol without replicas. To keep the discussion simple, we consider the case in which the VSM is accessed by two users; however, our case study can be generalised to an arbitrary number of users. We only give an informal description of the specification and the implementation we provide for VSMs, while we defer all formal definitions to [4], §B.

The Specification. In a virtual shared memory protocol, a distributed system provides to two or more users the ability to write and read memory locations which are physically stored at different nodes, while giving them the illusion that the whole memory is stored at a single node. 


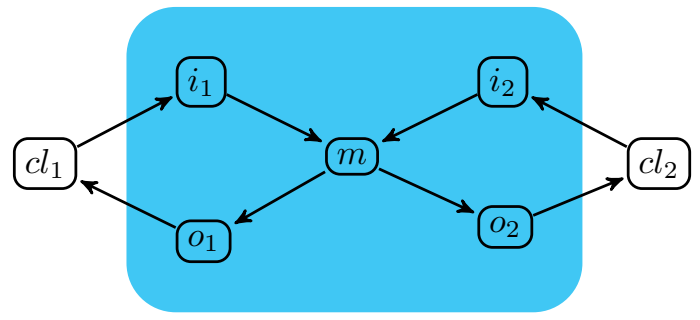

The system $\mathcal{V}$ specifying a VSM is depicted on the left. Nodes $i_{j}, o_{j}$, where $j=1,2$, regulate the flow of messages between a client $c l_{j}$ and the site where the memory is actually stored, $m$. Node $i_{j}$ discards any ill-formed request issued by client $c l_{j}$, while $o_{j}$ ensures that the answer by $m$ corresponds to a request originally performed by $c l_{j}$. More specifically, let $j=1,2$. Upon receiving a message of the form $\operatorname{read}(\mathrm{x})$, or write $(\mathrm{x}, n)$, node $i_{j}$ will store it in a local queue. At any given point, it will dequeue the next element stored and will forward it through channel $c_{j}$. Node $m$ is equipped with two queues, $q_{1}$ and $q_{2}$; upon receiving a request from node $i_{j}$, it will enqueue it in $q_{j}$. At any given point, node $m$ can dequeue the next request from one of the queues $q_{k}$, $k=1,2$. If such a request has the form read $\mathrm{x}$, then it will forward the value stored at its memory location $x$ along channel $d_{k}$. If the request has the form write $(\mathrm{x}, n)$, node $m$ will update the content of variable $\mathrm{x}$ to $n$, then it will broadcast the new value of $\mathrm{x}$ along channel $d_{k}$. Upon receiving a message along channel $d_{k}$, node $o_{k}$ stores it in a local queue. At any given point, it non-deterministically dequeues the next stored element, and forwards it to client $c l_{k}$.

Since our proof methods are sound only for finite spanning systems, we can assume that the size of the memory stored at node $m$ is finite, since the nodes $c l_{j}, j=1,2$ can only send a finite amount of requests to the system $\mathcal{V}$.

The Implementation. Next we turn our attention to a possible distributed implementation of our VSM. The idea is that of partitioning the memory stored at node $m$ of the specification, among different nodes, $m_{1}, \cdots, m_{k}$.

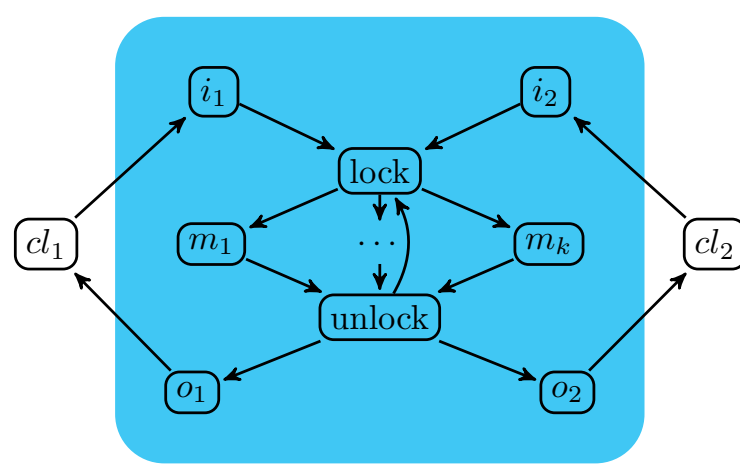

Each node contains only a subset of the locations stored in the total memory, and no memory location is stored in more than one node. To ensure that requests are processed in the same order in which they have been issued by a client, the access to nodes $m_{1}, \cdots, m_{k}$ is regulated by two nodes, called lock and unlock. These two nodes ensure that nodes $m_{1}, \cdots, m_{k}$ never handle two requests concurrently.

In the implementation $\mathcal{I}$, the behaviour of nodes $c l_{j}, i_{j}$ and $o_{j}$, where $j=1,2$, is the same as in the specification $\mathcal{V}$. Therefore we concentrate on describing the behaviour of the other nodes. Node lock is equipped with two queues $q_{1}, q_{2}$. Upon receiving a request along channel $c_{j}, j=1,2$, it will store it in the queue $q_{j}$. Node lock is also equipped with a boolean flag $l$, initially set to true. At any 
given time, node lock checks if $l$ is set to true; in this case, it sets the flag to false, then it selects a queue $q_{k}, k=1,2$, dequeues the next message stored in it, and broadcasts it along channel $c_{k}$. Node lock will then wait to receive a message along channel $d_{k}$, before resetting the flag $l$ to false. Any message broadcast by node lock along channels $d_{k}$ will be received by all nodes $m_{1}, \cdots, m_{k}$. Such a message contains either a request to read and write some memory location $\mathrm{x}$, which is stored in exactly one of such nodes, say $m_{n}$. Node $m_{n}$ will reply to this request, updating the contents of location $\mathrm{x}$, if needed, by broadcasting a message along channel $d_{k}$, which will in turn received by node unlock. Upon receiving a message along channel $d_{k}$, node unlock immediately broadcasts it along the same channel, causing both the message to be delivered at node $o_{k}$, and node lock to set its internal flag to true.

In $4, \S \mathrm{B}$, we give a formal description in our language of both the Specification, as the system $\mathcal{V}$, and the distributed Implementation, as the system term $\mathcal{I}$. The proof that the Implementation satisfies the Specification consists in establishing that $\mathcal{I} \simeq \mathcal{V}$. This is achieved by using the alternative characterisations of the preorders in terms of traces and Deadlock traces. A more detailed explanation of both the specification and implementation of our virtual shared memory can be found in [2, $\S 5.5$ at Page 124 .

Theorem 4. $\operatorname{Dtraces}(\mathcal{I})=\operatorname{Dtraces}(\mathcal{V})$ and $\operatorname{Traces}(\mathcal{I})=\operatorname{Traces}(\mathcal{V})$. In particular, $\mathcal{I} \simeq \mathcal{V}$.

\section{Conclusions}

The achievements of this paper have been more theoretical than practical, although we have also provided some evidence of applicability, via a case study. Many other applications can be found in Chapter 5 of [2]; these include connectionless and connection-oriented routing, for which implementations at different levels of the TCP/IP reference model have been provided, and multicast routing.

To the best of our knowledge, this paper presents the first completeness result for testing preorders applied to distributed systems. The proof is non-trivial, requiring the isolation of our extensional actions, and the detailed programming of the characteristic testing contexts. These probe systems via the extensional actions and then combine the results of these probes to elicit behavioural characteristics. The ability to define these characteristic tests also depends on the level of our abstraction of our system descriptions. It is far from clear if the completeness result remains true at other levels of abstraction, or if more general systems are considered. For example we already know from [3] that if we add probabilistic behaviour to nodes then the natural generalisation to probabilistic simulations no longer characterises the (probabilistic) testing preorders. Indeed it was this surprising phenomenon which prompted the research reported in the current paper. So it will be interesting to see what extra constructs can be added to our current non-probabilistic calculus without invalidating our characterisation results. Possibilities include introducing features which can be found 
in wireless networks, such as node mobility [19]16]9], or introducing time and/or collisions, as in 13/5 11|2111.

\section{References}

1. M. Bugliesi, L. Gallina, A. Marin, S. Rossi, and S. Hamadou. Interference-sensitive preorders for manets. In QEST, pages 189-198, 2012.

2. A. Cerone. Foundations of Ad Hoc Wireless Networks. PhD thesis, Trinity College Dublin, 2012. http://software.imdea.org/ andrea.cerone/works/thesis.pdf

3. A. Cerone and M. Hennessy. Modelling probabilistic wireless networks. LMCS, $9(3), 2013$.

4. A. Cerone and M. Hennessy. Characterising testing preorders for broadcasting distributed systems (extended version), 2014. http://software.imdea.org/ andrea.cerone/works/TGC14extended.pdf.

5. Andrea Cerone, Matthew Hennessy, and Massimo Merro. Modelling mac-layer communications in wireless systems (extended abstract). In COORDINATION, pages 16-30, 2013.

6. Conrado Daws, Marta Z. Kwiatkowska, and Gethin Norman. Automatic verification of the ieee-1394 root contention protocol with kronos and prism. Electr. Notes Theor. Comput. Sci., 66(2):104-119, 2002.

7. R. De Nicola and M. Hennessy. Testing equivalences for processes. TCS, 34:83-133, 1984.

8. C.F. Ene and T. Muntean. Testing theories for broadcasting processes. Sci. Ann. Cuza Univ, 11:214-230, 2002.

9. F. Ghassemi, W. Fokkink, and A. Movaghar. Equational reasoning on mobile ad hoc networks. Fund. Inf., 105(4):375-415, 2010.

10. M. Hennessy and J. Rathke. Bisimulations for a calculus of broadcasting systems. TCS, 200, 1998.

11. I. Lanese and D. Sangiorgi. An operational semantics for a calculus for wireless systems. TCS, 411(19):1928-1948, 2010.

12. Flavio Lerda and Riccardo Sisto. Distributed-memory model checking with spin. In SPIN, pages 22-39, 1999.

13. M. Merro, F. Ballardin, and E. Sibilio. A timed calculus for wireless systems. TCS, 412(47):6585-6611, 2011.

14. R. Milner. A calculus of communicating systems. LNCS, 92, 1980.

15. Madanlal Musuvathi and Dawson R. Engler. Model checking large network protocol implementations. In NSDI, pages 155-168, 2004.

16. S. Nanz and C. Hankin. A framework for security analysis of mobile wireless networks. TCS, 367, 2006.

17. Sebastian Nanz and Chris Hankin. Static analysis of routing protocols for ad-hoc networks, March 252004.

18. K. Prasad. A calculus of broadcasting systems. SCP, 25, 1995.

19. A. Singh, C.R. Ramakrishnan, and S.A. Smolka. A process calculus for mobile ad hoc networks. SCP, 75(6):440-469, 2010.

20. Andrew S Tanenbaum. Computer Networks. 4th Ed. Prentice Hall PTR, 2002.

21. M. Wang and Y. Lu. A timed calculus for mobile ad hoc networks. arXiv preprint arXiv:1301.0045, 2013. 